\title{
Effectiveness of intramuscular gluteal glucocorticoid injection versus intra- articular glucocorticoid injection in knee osteoarthritis: design of a multicenter randomized, 24 weeks comparative parallel-group trial
}

Marianne F. Mol ${ }^{1}$, Jos Runhaar ${ }^{1 *}$, P. Koen Bos², Desirée M. J. Dorleijn ${ }^{3}$, Marijn Vis ${ }^{4}$, Jacobijn Gussekloo, ${ }^{5,6}$, Patrick J. E. Bindels ${ }^{1}$ and Sita M. A. Bierma-Zeinstra ${ }^{1,2}$

\begin{abstract}
Background: The knee is symptomatically the most frequent affected joint in osteoarthritis and, in the Netherlands and other Western countries, is mainly managed by general practitioners (GPs). An intra-articular glucocorticoid injection is recommended in (inter) national guidelines for patients with knee osteoarthritis as an option for a flare of knee pain and/or for those who are not responding well to pain medication. An innovative approach that could replace the intra-articular injection is an intramuscular gluteal glucocorticoid injection. An intramuscular injection is easier to perform than an intra-articular injection with lesser risk of severe local adverse reactions. We hypothesize that intramuscular gluteal glucocorticoid injection is non-inferior in reducing knee pain compared to intra-articular glucocorticoid injection, with potentially a longer lasting effect than intra-articular injection.
\end{abstract}

Methods/design: The study will be a pragmatic randomized controlled non-inferiority trial with two parallel groups. A total of 140 patients aged 45 years and older with knee osteoarthritis who contacted their general practitioner and have persistent knee pain (score $\geq 3$ on $0-10$ numerical rating scale; $0=$ no knee pain) will be included.

Patients will be randomly allocated (1:1) to an injection of $40 \mathrm{mg}$ triamcinolone acetonide intra-articular in the knee joint or intramuscular in the ipsilateral ventrogluteal area.

The effect of treatment will be evaluated by questionnaires at 2, 4, 8, 12, and 24 weeks after injection. The primary outcome is patients' reported severity of knee pain measured with the pain subscale of the Knee injury and Osteoarthritis Outcome Score 4 weeks after injection. Statistical analysis will be based on both the per-protocol and the intention-to-treat principle.

* Correspondence: j.runhaar@erasmusmc.nl

'Department of General Practice, Erasmus MC University Medical Center Rotterdam, PO-box 2040, 3000, CA, Rotterdam, The Netherlands

Full list of author information is available at the end of the article

(c) The Author(s). 2020 Open Access This article is licensed under a Creative Commons Attribution 4.0 International License, which permits use, sharing, adaptation, distribution and reproduction in any medium or format, as long as you give appropriate credit to the original author(s) and the source, provide a link to the Creative Commons licence, and indicate if changes were made. The images or other third party material in this article are included in the article's Creative Commons licence, unless indicated otherwise in a credit line to the material. If material is not included in the article's Creative Commons licence and your intended use is not permitted by statutory regulation or exceeds the permitted use, you will need to obtain permission directly from the copyright holder. To view a copy of this licence, visit http://creativecommons.org/licenses/by/4.0/ The Creative Commons Public Domain Dedication waiver (http://creativecommons.org/publicdomain/zero/1.0/) applies to the data made available in this article, unless otherwise stated in a credit line to the data. 


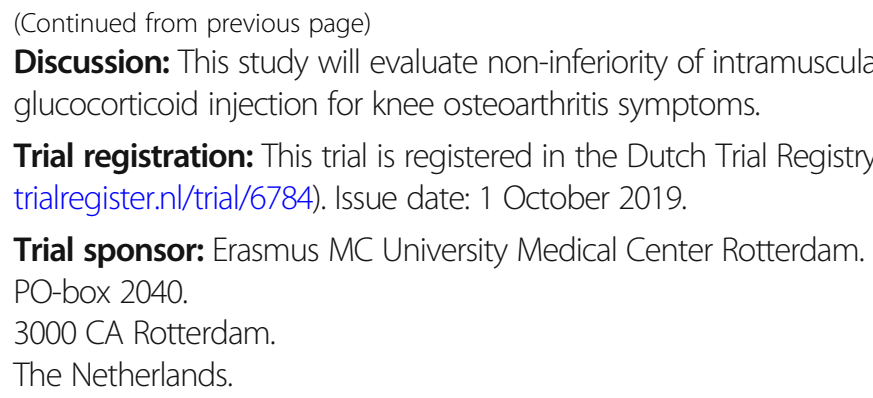

Keywords: Knee osteoarthritis, Glucocorticoid injection, Intra-articular, Intramuscular, Randomized controlled trial, Noninferiority, Primary care, General practice

\section{Background}

The knee is symptomatically the most frequent affected joint in osteoarthritis (OA). In the Netherlands and other Western countries this is mainly managed by general practitioners [1]. The prevalence of knee OA in general practice was estimated around 40.2 per 1000 patient years (28.9 men; 51.4 women) in 2018 [2].

For OA patients, pain and disability are the most important reasons to seek care of a health professional [35]. If patients are not responding satisfactorily to paracetamol, NSAIDs and non-drug treatment, or in cases of interim aggravation, the evidence-based guideline from the Dutch College of General Practitioners on 'Nontraumatic knee complaints' suggests intra-articular (IA) glucocorticoid injection with 20 to $40 \mathrm{mg}$ triamcinolone acetonide [6]. In several international guidelines IA glucocorticoid injection is also recommended for these abovementioned indications $[7,8]$.

Despite the long-standing frequent application of IA glucocorticoids, there is an ongoing debate about their effectiveness and safety $[9,10]$. IA glucocorticoid injection in patients with knee OA leads to a moderate improvement in pain, but only in the short term (1 to 6 weeks after injection) [9]. IA injection has a small risk of the serious adverse reaction of septic arthritis on the short term [11]. In recent literature there is controversy over the chondrotoxicity of IA glucocorticoids on the longer term [12-14].

An additional obstacle in the way of IA injection is that GPs might feel incompetent to administer this type of injection due to lack of training and experience [15]. Due to the GP's restraint, knee OA patients who could benefit from IA injection might not always receive timely injection [15].

Intramuscular (IM) glucocorticoid injection could be a valuable alternative treatment for IA glucocorticoid injection for patients with knee OA. IM administration eliminates the risk of septic arthritis and direct cartilage toxicity. The favorable effect of IM glucocorticoids on musculoskeletal pain has been studied originally in patients with rotator cuff disease and is used for rheumatoid arthritis $[16,17]$. In a recent study from our study group, a clinical relevant and statistical significant difference in pain reduction was found for IM glucocorticoid injection compared to placebo in patients with hip OA [18]. Remarkably, the clinically relevant effect of the IM injection lasted at least 12 weeks. As of now no direct comparison between the effectiveness of IM and IA glucocorticoid injection in knee OA has been made.

We will perform a randomized controlled twoparallel-groups trial in patients with knee OA included from general practices, assessing the non-inferiority of an IM gluteal glucocorticoid injection compared to an IA glucocorticoid knee injection at 4 weeks follow-up. We hypothesize that IM gluteal glucocorticoid injection is non-inferior to IA glucocorticoid injection in reducing knee pain 4 weeks after injection, with potentially a longer lasting effect for at least 12 weeks.

\section{Primary objective}

The primary objective is to assess whether IM gluteal glucocorticoid injection is non-inferior to IA knee glucocorticoid injection in reducing knee pain, measured with the Knee injury and Osteoarthritis Outcome Score (KOOS) pain subscale, in patients with knee OA in general practice at 4 weeks after injection.

\section{Secondary objectives}

The study will evaluate the differences in reported adverse events frequency and co-interventions of patients allocated to an IM gluteal glucocorticoid injection or to an IA glucocorticoid injection. Differences between the two treatment groups in several outcome measures related to knee recovery, on short and longtime follow-up (2-24 weeks) and quality of life will be measured (see Table 2).

\section{Methods}

Design

This study is a pragmatic randomized controlled noninferiority trial with two parallel groups with a follow-up of 24 weeks (see Fig. 1). The Medical Ethics Committee 
of Erasmus MC University Medical Center Rotterdam approved this trial (MEC 2017-563). Any modifications to the protocol which may impact on the conduct of the study, potential benefit of the patient or may affect patient safety, including changes of study objectives, study design, patient population, sample sizes, study procedures, or significant administrative aspects will require a formal amendment to the protocol. Such amendment will be approved by the Medical Ethics Committee of Erasmus MC University Medical Center Rotterdam prior to implementation. All patients will give written informed consent prior to data collection.

\section{Patient selection}

Patients with OA of the knee will be recruited by participating GPs located in the South-West of the Netherlands. Patient selection can take place in two different ways. The electronic patient files will be scanned for the International Classification of Primary Care (ICPC) codes L90 (knee OA) and L15 (knee symptoms/complaints) in order to identify eligible patients who have no contraindications for participation in this study. See Table 1 for the inclusion- and exclusion criteria. Patients who are identified with the ICPC code L15 are only selected if there is a note by the GP or a radiology report that mentions 'osteoarthritis' or 'cartilage degeneration'.

The second way is that GPs are asked to invite patients who consult them for knee OA to participate in the study. The GPs are asked to screen the inclusion- and exclusion criteria for all patients and will also directly assess whether there is an indication for glucocorticoid injection. An intra-articular glucocorticoid injection is recommended in guidelines for patients with knee osteoarthritis as an option for a flare of knee pain and/or for those who are not responding well to pain medication. Patients who have had an IA injection in the knee during the previous 6 months will be excluded, since a prolonged treatment effect of 24 weeks after injection has been described [19]. Patients with diabetes mellitus on insulin therapy or with a poor glycemic control (as

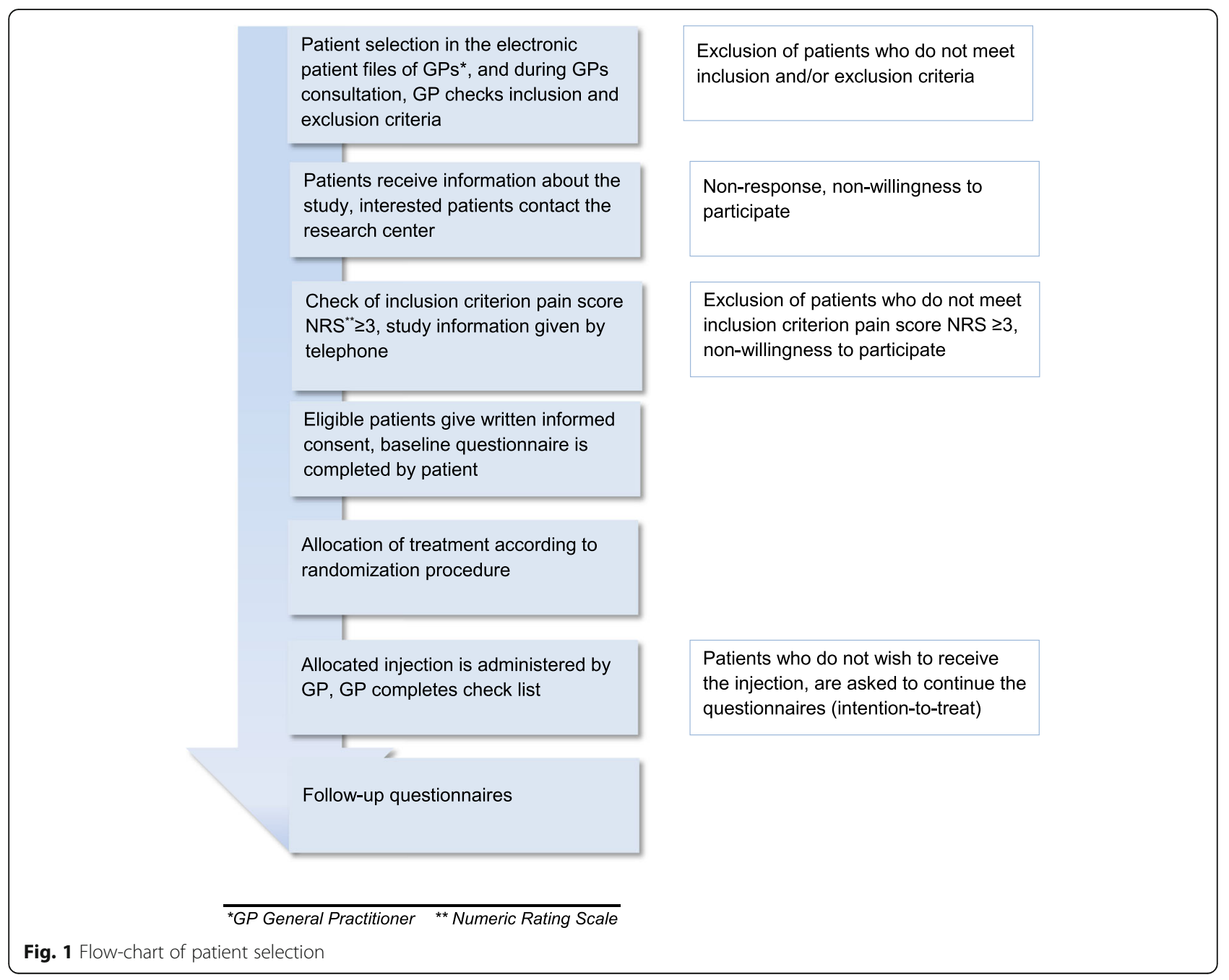


Table 1 Eligibility criteria

Inclusion criteria:
1) contacted their GPa (consultation and/or repeat pain medication
prescription) due to knee $O A^{b}$ (ICPC $L 90$ or $\left.L 15\right)$ during the past 5
years;
2) aged 45 years and over;
3) symptomatic knee OA for at least 3 months prior to enrolment;
4) a minimum score of 3 on the NRS ${ }^{\mathrm{d}}$ asking about the severity of knee
pain averaged over the past week $(0-10 ; 0=$ no knee pain);
5) glucocorticoid injection is indicated in this patient

\section{Exclusion criteria:}

1) use of oral glucocorticoids;

2) intra-articular injection in a knee in the previous 6 months;

3) allergy to glucocorticoids;

4) local or systemic infection, recent vaccination with live attenuated vaccine;

5) type 1 diabetes mellitus, type 2 diabetes mellitus on insulin therapy, poorly controlled type 2 diabetes mellitus;

6) presence of inflammatory rheumatic diseases (such as rheumatoid arthritis, psoriatic arthritis, spondylartropathies);

7) coagulopathy, use of anticoagulants, use of dual antiplatelet therapy;

8) a history of gastric/duodenal ulcer or a present gastric/duodenal ulcer;

9) currently receiving care of an orthopaedic surgeon for OA of the hip and/or knee;

10) incapacity to complete questionnaires in Dutch;

11) incapacity to give informed consent.

aGP General Practitioner ${ }^{\mathrm{b}} \mathrm{OA}$ Osteoarthritis ${ }^{\mathrm{C}} \mathrm{ICPC}$ International Classification of Primary Care ${ }^{\mathrm{d}}$ Numeric Rating Scale

assessed by their GP) cannot participate, as they might be at risk of prolonged blood glucose level elevation after glucocorticoid injection [20, 21]. Patients who have been referred to an orthopedic surgeon will also be excluded from participation considering that these patients could become candidates for total knee or hip arthroplasty during the follow-up period of the study. The risk of periprosthetic joint infection (PJI) is increased in patients who received an IA glucocorticoid injection in the 3 months prior to arthroplasty [22, 23]. It is not known if IM glucocorticoid injection increases the risk of PJI.

For patients who are selected via their electronic patient file, the actual amount of knee pain they experience is not known by their GP. These patients' knee pain level will be checked over the phone by the researchers (given that the patient is willing to participate in the study, see Fig. 1). A minimum score of 3 on the numerical rating scale (NRS, $0-10 ; 0=$ no knee pain) is required in order to participate in the trial. If a patient has an NRS knee pain score $<3$, the patient will be asked to contact the research team in case of future increase in knee pain. In case a patient has bilateral knee OA, the most painful knee is selected as the 'study knee'.

\section{Procedures}

The GPs will inform all eligible patients about the study in writing. Patients will receive information about the study and a reply card. Once the research team receives a reply card with a positive response from a patient, a researcher will contact this patient by telephone. The researcher will ask the patient about the severity of knee pain averaged over the past week. To all patients with a pain score $\geq 3$ additional written information about the study will be sent. Some days later, the researcher or trained research assistant will contact the patients again to further explain the study and to answer remaining questions. Patients who are interested to participate will be asked to give written informed consent. After the patients have given this consent, the baseline questionnaire is sent to these patients.

After completion of the baseline questionnaire, the patient will be randomly allocated to one of the two treatment groups. The GP and the patient will be informed about the outcome of randomization. The GP will prepare and administer the allocated injection. We aim to have the injection administered within 1 week after completion of the baseline questionnaire. This is to ascertain the pain score at the moment of injection is unchanged or close to the baseline score. Change in pain since baseline can never lead to exclusion of a trial participant.

The GP will complete a case report form at the patients' visit for administration of the injection. This report form asks for the American College of Rheumatology criteria for clinical diagnosis of knee OA, location of injection, severity of knee pain averaged over the past week and the batch number of the triamcinolone acetonide [24].

All patients will be referred for an AP weightbearing X-ray of the studied knee if an X-ray has not been made in the 12 months prior to enrolment. The 12 month period was chosen as the risk of annual radiographic OA progression by at least one KellgrenLawrence (K-L) grade has been estimated low [25]. Therefore, the X-ray does not have to be obtained directly at baseline, but will be made during followup. We consider the radiograph necessary in order to facilitate comparison between our study population and patient data collected in previous studies. Two researchers will independently assess the X-rays to grade radiographic knee $\mathrm{OA}$, using the $\mathrm{K}-\mathrm{L}$ classification [26].

\section{Randomization}

After the patients sign informed consent, they will be randomized and receive their allocated intervention. After informed consent the patient will be assigned a unique trial number.

An independent researcher, who will not meet or contact the patients, has prepared a computer generated randomization list using 1:1 allocation and random blocks of 8,6 or 4 in order to ensure concealment of allocation. 


\section{Blinding}

Due to the pragmatic nature of this trial, the patient and the GP are not blinded for treatment allocation. The researcher involved in data analysis will be blinded for treatment allocation.

\section{Intervention}

The investigational treatment will consist of $40 \mathrm{mg}$ triamcinolone acetonide (Kenacort-A 40). The chosen dosage of glucocorticoid is based on clinical experience [27]. No local anesthetic will be added to the injection. The GP will inject either IA in the knee joint or IM in the ipsilateral ventrogluteal region. All participating GPs will be invited for an optional IA knee injection training under supervision of an experienced orthopedic surgeon (PKB).

The superolateral IA injection approach to the knee will be used (just below the upper border of the patella and $1 \mathrm{~cm}$ lateral to the lateral border of the patella, see Fig. 2b). This approach has an accuracy of $91 \%$ for needle placement in the IA space of the knee and is recommended by the Dutch College of GPs [6, 28].

The IM injection will be administered in the ventrogluteal region (the region between the iliac crest, greater trochanter of the femur and anterior iliac spine, see Fig. 2a) in order to prevent injury to the sciatic nerve $[29,30]$. Moreover, administration in the ventrogluteal region diminishes the possibility of subcutaneous injection in overweight patients since the layer of subcutaneous fat is less thick in the ventrogluteal region compared to the dorsogluteal region [31]. It is well known that knee OA is more common in patients with a BMI $>27 \mathrm{~kg} / \mathrm{m}^{2}$ [32].

Co-interventions are allowed during the follow-up and will be monitored.

\section{Measurements}

\section{Baseline measurements}

See Table 2 for an overview of baseline measurements. Demographic measurements consist of age, sex, educational level, and daily occupation. Also self-reported length and weight are reported. Medication use for knee OA will be measured using a multiple choice format where patients can select multiple answers and add a missing medicament.

Intermittent and constant OA pain will be measured with the Intermittent and Constant OsteoArthritis Pain score (ICOAP:0-100; $0=$ no pain) [33]. Knee complaint characteristics (duration of symptoms at baseline, sensation of swelling in the knee as an indicator of flare-up) will be recorded. Knee pain severity averaged over the last week will be measured with an 11-point numerical rating scale (NRS:0-10;0 = no pain). Health related Quality of Life (QoL) will be measured with the EQ-5D$5 \mathrm{~L}$ (scores ranging from $-0.446=$ worst health related QoL to $1.0=$ perfect health related QoL) [34, 35] Comorbidity will be measured at baseline using a multiple choice format where patients can select multiple answers and add a missing comorbid disease. Also measured at baseline will be physical activity over the past week (IPAQ short), neuropathic pain (modified painDETECT questionnaire), patients' preferred injection site (knee or ventrogluteal area) and patients' expected treatment response [36, 37].

\section{Follow-up measurements}

Outcomes are measured at 2, 4, 8, 12 and 24 weeks after administration of the injection using digital questionnaires. Patients without an electronic mailbox will receive paper questionnaires. The primary outcome is patient reported severity of pain at 4 weeks after injection measured with the KOOS pain subscale $(0-100 ; 0=$

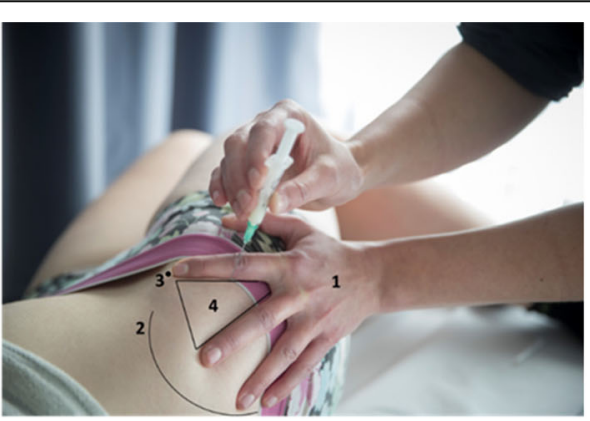

A.

1: palm of hand on greater trochanter

2: iliac crest

3: anterior superior iliac spine

4: area for injection

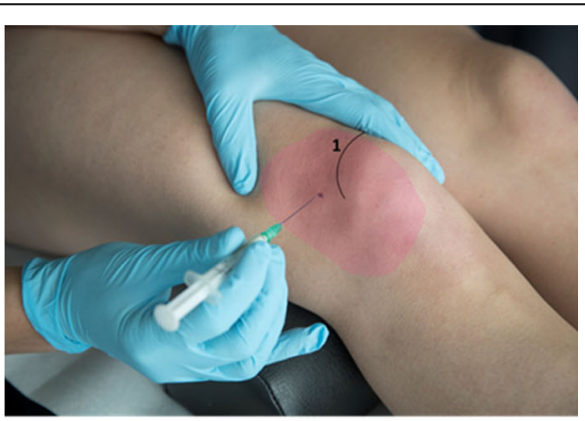

B.

1: upper border of the patella

Fig. 2 Ventrogluteal intramuscular and superolateral intra-articular injection techniques 
Table 2 Scheduled measurements of primary and secondary outcomes

\begin{tabular}{|c|c|c|c|c|c|c|c|}
\hline Measurement & Baseline & Injection & 2 weeks fu & 4 weeks fu & 8 weeks fu & 12 weeks fu & 24 weeks fu \\
\hline \multicolumn{8}{|l|}{ Primary outcome measure } \\
\hline KOOS pain subscale & $x$ & & $x$ & $x$ & $x$ & $x$ & $x$ \\
\hline \multicolumn{8}{|l|}{ Secondary outcome measures } \\
\hline Adverse events & & & $x$ & & & & \\
\hline Hospitalization & & & $x$ & $x$ & $x$ & $x$ & $x$ \\
\hline Co-interventions (iMCQ) & & & $x$ & $x$ & $x$ & $x$ & $x$ \\
\hline Medication use for knee OA & $x$ & & $x$ & $x$ & $x$ & $x$ & $x$ \\
\hline Re-injection with glucocorticoid & & & & & $x$ & $x$ & $x$ \\
\hline KOOS stiffness & $x$ & & $x$ & $x$ & $x$ & $x$ & $x$ \\
\hline KOOS function in daily living & $x$ & & $x$ & $x$ & $x$ & $x$ & $x$ \\
\hline KOOS sports and recreation & $x$ & & $x$ & $x$ & $x$ & $x$ & $x$ \\
\hline KOOS QoL & $x$ & & $x$ & $x$ & $x$ & $x$ & $x$ \\
\hline ICOAP & $x$ & & $x$ & $x$ & $x$ & $x$ & $x$ \\
\hline OMERACT OARSI responder criteria & & & $x$ & $x$ & $x$ & $x$ & $x$ \\
\hline Knee pain over past week (NRS) & $x$ & $x$ & $x$ & $x$ & $x$ & $x$ & $x$ \\
\hline Perceived recovery (Likert scale) & & & $x$ & $x$ & $x$ & $x$ & $x$ \\
\hline Knee complaint characteristics & $x$ & & $x$ & $x$ & $x$ & $x$ & $x$ \\
\hline Health related QoL & $x$ & & & & & & \\
\hline \multicolumn{8}{|l|}{ Additional measurements } \\
\hline Radiograph of study knee & & & & & & $x$ & \\
\hline Check of ACR criteria & & $x$ & & & & & \\
\hline Painfulness of injection (NRS) & & & $x$ & & & & \\
\hline Demographic information & $x$ & & & & & & \\
\hline Co-morbidity & $x$ & & & & & & \\
\hline Physical activity over the past week (IPAQ short) & $x$ & & & & & & \\
\hline Neuropathic pain (Modified painDETECT Questionnaire) & $x$ & & & & & & \\
\hline Patients' preferred injection site & $x$ & & & & & & \\
\hline Patients' expected treatment response & $x$ & & & & & & \\
\hline
\end{tabular}

extreme pain). Secondary study endpoints are listed in Table 2. Patients' perceived recovery is measured with a 7-point Likert scale that will be dichotomized in recovered ('complete recovery', 'much improved', 'slightly improved') and not-recovered ('no change', 'slightly worse', 'much worse',"worse than ever') [18]. Percentage responders is defined by the OMERACTOARSI criteria: High improvement $(\geq 50 \%)$ in KOOS pain subscale or in KOOS function in daily living subscale and absolute increase $\geq 20$ points in KOOS pain subscale or function in daily living subscale, if not then improvement in at least 2 of the 3 following domains: 1$) \geq 20 \%$ improvement in KOOS pain subscale and $\geq 10$ points increase in KOOS pain subscale, $2) \geq 20 \%$ improvement in KOOS function in daily living subscale and $\geq 10$ points increase in KOOS function in daily living subscale, 3) $\geq 20 \%$ increase in global score and $\geq 10$ points increase in global score.
In this study patients' global score will be measured with a patients' perceived recovery score measured on a 7-point Likert scale. This domain is considered improved if a patient fills in 'complete recovery', 'much improved', or 'slightly improved' [38]. Two weeks after administration of the injection patients are asked to report adverse events. Also, follow-up questionnaires at all time points ask about hospitalization to monitor Serious Adverse Events. Co-interventions including medication, non-drug therapies such as physiotherapy, referrals and surgery will be measured with the modified medical consumption questionnaire of the Institute for Medical Technology Assessment (iMCQ) [39]. Experienced painfulness of injection will be registered 2 weeks after glucocorticoid injection. All data will be handled according to the Data Monitoring Plan, as drafted and approved by the funder in preparation of data collection. 


\section{Sample size}

For the sample size calculation, we used data from the study of Henriksen et al. that evaluated the clinical benefits of an IA glucocorticoid injection given before exercise therapy in patients with knee OA [40]. The results of the study reported a baseline standard deviation of 16 for the KOOS pain (recommended by the KOOS for sample size calculations is a standard deviation of 15). The minimal important difference (=non-inferiority margin) between both treatment groups of the patient reported outcome KOOS (0-100) was set at 7 points (effect size of 0.44) [41, 42].

For the non-inferiority of an IM gluteal glucocorticoid injection compared to an IA knee glucocorticoid injection, we will need 65 patients per group, using a power of $80 \%$, an alpha of $5 \%$, a non-inferior margin of 7 and a SD of 16. Taking into account a loss to follow-up of $5 \%$, this trial needs to include $(2 * 65)+(0.05 * 2 * 65)=\sim 140$ patients. We expect a low percentage of loss to followup because of the relative short follow-up period of 24 weeks and our prior experience in glucocorticoid trials $[18,43]$.

\section{Data analyses}

Imbalance in the baseline variables of the two treatment arms might occur after randomization. This is problematic if the imbalanced variable is related to the outcome variable, as this could lead to confounding [44, 45]. In case imbalance occurs, we will adjust for relevant variables. Descriptive statistics will be used to describe patients' and complaints characteristics at baseline. Analyses will be adjusted for variables that hamper the baseline interchangeability of groups when there are clinically relevant differences between groups of over $10 \%$.

The primary outcome is patient reported severity of pain at 4 weeks after injection measured with the KOOS pain $(0-100 ; 0=$ extreme pain). We will use a noninferiority design to assess if an IM gluteal injection is non-inferior to an IA knee injection with regard to this outcome. In non-inferiority comparisons intention-totreat analysis can bias towards the null and could increase type I error; the risk of falsely claiming noninferiority [46]. Therefore, the per-protocol analysis will be the primary analysis. Included in the per-protocol analysis will be patients who received the assigned injection and reported the KOOS pain at 4 weeks follow-up. In case a patient from the IM injection group receives an additional IA injection earlier than 6 weeks after the study injection, this will be considered as a protocol violation and the patient will be excluded from the perprotocol analysis. The reason for this is that the guideline from the Dutch College of General Practitioners recommends to leave at least 6 weeks between two consecutive injections [6]. Non-inferiority of the IM injection will also be assessed using both intention-to-treat and per-protocol analysis at 2, 8, 12 and 24 weeks follow-up and for the outcome KOOS pain. For the other outcome measures we will calculate mean differences.

We expect $10-15 \%$ of missing data (incompletely filled in paper questionnaires and loss to follow-up). We will contact the patients to pose them the missing questions again in order to minimize missing data for the primary outcome. Multiple imputations will be performed for missing values (incompletely filled in questionnaires), creating at least five imputed datasets.

Linear mixed models with repeated measures will be used to calculate group differences over time for the primary outcome as this is a continuous variable. To model the covariance of repeated measures by patients, a structure will be chosen with the lowest Akaike's information criterion. Fixed effects will be time, and time by treatment.

Linear mixed models with repeated measures will also be used for the continuous secondary outcomes: KOOS, NRS, WOMAC, ICOAP, and EQ-5D-5 L. Generalized estimating equations analyses with repeated measures will be performed for the dichotomous outcomes: patients' perceived recovery (7 point Likert scale), and the OMERACT-OARSI responder criteria. Before generalized estimating equations analyses, multiple imputations will be performed for missing values of secondary study parameters, creating at least five imputed datasets.

When patients underwent a total knee replacement surgery, data of these patients will be included up to the date of surgery. Missing data for secondary outcome measurements will be handled similarly as missing data for the primary outcome.

\section{Subgroup analysis}

An explorative, pre-defined, subgroup analysis will be performed assessing the interaction effects between injections regarding the severity of knee pain at baseline (NRS pain score of $>=7$ versus $<7$ ) on the primary outcome [47]. Generalized estimating equations will be used to analyze differences between groups concerning adverse events, medical consumption and medication usage.

\section{Discussion}

This study will evaluate non-inferiority of intramuscular glucocorticoid injection compared to intra-articular glucocorticoid injection for knee osteoarthritis symptoms, during 24 weeks of follow-up. The primary outcome is the patient reported severity of pain at 4 weeks after injection, using a non-inferiority margin of 7 points 
on the patient reported outcome KOOS between both treatment groups.

The sample size calculations indicated a required sample of 140 participants, taking into account a loss to follow-up of $5 \%$. Recruitment was finalized on February $11,2020$.

\section{Strength and limitations}

The current study is a high-quality pragmatic trial, which will provide a reliable comparison between intraarticular and intramuscular corticosteroid injections for individuals with knee OA and will make implementation of its results highly feasible. Obviously, patients will not be blinded to the location of the injection in the current study. Although this might bias the results towards the by the patients preferred injection location (patients' preference will be assessed), we already know from our previous study on hip OA [18] that the intramuscular corticosteroid injection was superior to placebo. So the clinical effectiveness of the intramuscular corticosteroid injection did not rely on contextual effects only.

\section{Abbreviations}

GP: General Practitioner; IA: intra-articular; ICOAP: Intermittent and Constant OsteoArthritis Pain; ICPC codes: International Classification of Primary Care (ICPC) codes; IM: Intramuscular; iMCQ: Institute for Medical Technology Assessment; K-L grade: Kellgren-Lawrence grade; KOOS: Knee injury and Osteoarthritis Outcome Score; NRS: numerical rating scale; OA: Osteoarthritis; PJI: periprosthetic joint infection; QoL: Quality of Life

\section{Acknowledgements}

Not applicable.

\section{Authors' contributions}

SMABZ, PKB, MV, JG, PJEB and DMJD participated in the design of the study. $S M A B Z, M F M$ and JR coordinate the trial and are responsible for data collection. MFM and JR prepared the article. All authors have read and approved the final manuscript.

\section{Funding}

Financial support was received from ZonMw (The Netherlands Organisation for Health Research and Development). This funding source had no role in the design of this study and will not have any role during its execution, analyses, interpretation of the data, or decision to submit results.

\section{Availability of data and materials}

Not applicable.

\section{Ethics approval and consent to participate}

This study was approved by the Medical Ethics Committee of Erasmus MC University Medical Center Rotterdam (MEC 2017-563). All participants will give written informed consent prior to data collection.

\section{Consent for publication}

Not applicable.

\section{Competing interests}

The authors declare that they have no conflict of interest.

\section{Author details}

'Department of General Practice, Erasmus MC University Medical Center Rotterdam, PO-box 2040, 3000, CA, Rotterdam, The Netherlands.

${ }^{2}$ Department of Orthopaedic Surgery, Erasmus MC University Medical Center Rotterdam, Rotterdam, The Netherlands. ${ }^{3}$ Department of Orthopaedic Surgery, University of Groningen, University Medical Center Groningen,
Groningen, The Netherlands. ${ }^{4}$ Department of Rheumatology, Erasmus MC University Medical Center Rotterdam, Rotterdam, The Netherlands. ${ }^{5}$ Department of Public Health and Primary Care, Leiden University Medical Center, Leiden, The Netherlands. ${ }^{6}$ Section of Gerontology and Geriatrics, Department of Internal Medicine, Leiden University Medical Center, Leiden, the Netherlands.

Received: 10 January 2020 Accepted: 31 March 2020

Published online: 11 April 2020

\section{References}

1. Oliveria SA, Felson DT, Reed I, Cirillo PA, Walker AM. Incidence of symptomatic hand, hip, and knee osteoarthritis among patients in a health maintenance organization. Arthritis Rheum. 1995;38(8):1134-41.

2. NIVEL: Incidence and prevalence knee osteoarthritis in general practice 2018. https://www.nivel.nl/nl/nivel-zorgregistraties-eerste-lijn/incidenties-enprevalenties. Accessed Sept 2019.

3. Paskins Z, Sanders T, Hassell AB. What influences patients with osteoarthritis to consult their GP about their symptoms? A narrative review. BMC Fam Pract. 2013:14:195

4. Rosemann T, Wensing M, Joest K, Backenstrass M, Mahler C, Szecsenyi J. Problems and needs for improving primary care of osteoarthritis patients: the views of patients, general practitioners and practice nurses. BMC Musculoskelet Disord. 2006;7:48.

5. Felson DT, Neogi T. Emerging Treatment Models in Rheumatology: Challenges for Osteoarthritis Trials. Arthritis Rheumatol. 2018;70(8):1175-81. https://doi.org/10.1002/art.40515. Epub 2018 Jun 6.

6. Dutch Association of General Practitioners: NHG-guideline Non-traumatic knee complaints (In Dutch). In.; 2016.

7. National Institute for Health and Care Excellence (NICE ): Osteoarthritis: care and management. https://www.nice.org.uk/guidance/cg177. Accessed Oct 2016.

8. Bannuru RR, Osani MC, Vaysbrot EE, Arden NK, Bennell K, Bierma-Zeinstra SMA, Kraus VB, Lohmander LS, Abbott JH, Bhandari M, et al. OARSI guidelines for the non-surgical management of knee, hip, and polyarticular osteoarthritis. Osteoarthr Cartil. 2019;27(11):1578-89.

9. Juni $P$, Hari R, Rutjes AW, Fischer R, Silletta MG, Reichenbach $S$, da Costa BR. Intra-articular corticosteroid for knee osteoarthritis. Cochrane Database Syst Rev. 2015;10:CD005328.

10. Liu SH, Dube CE, Eaton CB, Driban JB, McAlindon TE, Lapane KL. Longterm effectiveness of Intraarticular injections on patient-reported symptoms in knee osteoarthritis. J Rheumatol. 2018;45(9):1316-24.

11. Charalambous $C P$, Tryfonidis M, Sadiq S, Hirst P, Paul A. Septic arthritis following intra-articular steroid injection of the knee--a survey of current practice regarding antiseptic technique used during intra-articular steroid injection of the knee. Clin Rheumatol. 2003;22(6):386-90.

12. McAlindon TE, LaValley MP, Harvey WF, Price LL, Driban JB, Zhang M, Ward RJ. Effect of intra-articular triamcinolone vs saline on knee cartilage volume and pain in patients with knee osteoarthritis: a randomized clinical trial. JAMA. 2017;317(19):1967-75.

13. Zeng C, Lane NE, Hunter DJ, Wei J, Choi HK, McAlindon TE, Li H, Lu N, Lei G, Zhang $Y$. Intra-articular corticosteroids and the risk of knee osteoarthritis progression: results from the osteoarthritis initiative. Osteoarthr Cartil. 2019; 27(6):855-62.

14. Conaghan PG. Corticosteroids and osteoarthritis progression: a confounded issue. Osteoarthr Cartil. 2019;27(6):e5-6.

15. Liddell WG, Carmichael CR, McHugh NJ. Joint and soft tissue injections: a survey of general practitioners. Rheumatology (Oxford). 2005;44(8):1043-6.

16. Ekeberg OM, Bautz-Holter E, Tveita EK, Juel NG, Kvalheim S, Brox Jl. Subacromial ultrasound guided or systemic steroid injection for rotator cuff disease: randomised double blind study. BMJ. 2009;338:a3112.

17. Singh JA, Saag KG, Bridges SL Jr, AkI EA, Bannuru RR, Sullivan MC, Vaysbrot E, McNaughton C, Osani M, Shmerling RH, et al. 2015 American College of Rheumatology Guideline for the treatment of rheumatoid arthritis. Arthritis Care Res (Hoboken). 2016;68(1):1-25.

18. Dorleijn DMJ, Luijsterburg PAJ, Reijman M, Kloppenburg M, Verhaar JAN, Bindels PJE, Bos PK, Bierma-Zeinstra SMA. Intramuscular glucocorticoid injection versus placebo injection in hip osteoarthritis: a 12-week blinded randomised controlled trial. Ann Rheum Dis. 2018;77(6):875-82.

19. Arroll B, Goodyear-Smith F. Corticosteroid injections for osteoarthritis of the knee: meta-analysis. Bmj. 2004;328(7444):869. 
20. Waterbrook AL, Balcik BJ, Goshinska AJ. Blood glucose levels after loca musculoskeletal steroid injections in patients with diabetes mellitus: a clinical review. Sports Health. 2017;9(4):372-4.

21. Aleem AW, Syed UAM, Nicholson T, Getz CL, Namdari S, Beredjiklian PK, Abboud JA. Blood glucose levels in diabetic patients following corticosteroid injections into the subacromial space of the shoulder. Arch Bone Jt Surg. 2017:5(5):315-21.

22. Richardson SS, Schairer WW, Sculco TP, Sculco PK. Comparison of infection risk with corticosteroid or hyaluronic acid injection prior to Total knee Arthroplasty. J Bone Joint Surg Am. 2019;101(2):112-8.

23. Werner BC, Cancienne JM, Browne JA. The timing of Total hip Arthroplasty after Intraarticular hip injection affects postoperative infection risk. J Arthroplast. 2016;31(4):820-3.

24. Altman R, Asch E, Bloch D, Bole G, Borenstein D, Brandt K, Christy W, Cooke $T D$, Greenwald R, Hochberg M, et al. Development of criteria for the classification and reporting of osteoarthritis. Classification of osteoarthritis of the knee. Diagnostic and therapeutic criteria Committee of the American Rheumatism Association. Arthritis Rheum. 1986;29(8):1039-49.

25. Emrani PS, Katz JN, Kessler CL, Reichmann WM, Wright EA, McAlindon TE, Losina E. Joint space narrowing and Kellgren-Lawrence progression in knee osteoarthritis: an analytic literature synthesis. Osteoarthr Cartil. 2008;16(8): 873-82.

26. Kellgren JH, Lawrence JS. Radiological assessment of osteo-arthrosis. Ann Rheum Dis. 1957;16(4):494-502.

27. Shah A, Mak D, Davies AM, James SL, Botchu R. Musculoskeletal corticosteroid administration: current concepts. Can Assoc Radiol J. 2019; 70(1):29-36.

28. Hermans J, Bierma-Zeinstra SM, Bos PK, Verhaar JA, Reijman M. The most accurate approach for intra-articular needle placement in the knee joint: a systematic review. Semin Arthritis Rheum. 2011;41(2):106-15.

29. Mishra P, Stringer MD. Sciatic nerve injury from intramuscular injection: a persistent and global problem. Int J Clin Pract. 2010;64(11):1573-9.

30. Jung Kim H, Hyun Park S. Sciatic nerve injection injury. J Int Med Res. 2014; 42(4):887-97.

31. Larkin TA, Ashcroft E, Hickey BA, Elgellaie A. Influence of gender, BMI and body shape on theoretical injection outcome at the ventrogluteal and dorsogluteal sites. J Clin Nurs. 2018;27(1-2):e242-50.

32. Reijman M, Pols HA, Bergink AP, Hazes JM, Belo JN, Lievense AM, BiermaZeinstra SM. Body mass index associated with onset and progression of osteoarthritis of the knee but not of the hip: the Rotterdam study. Ann Rheum Dis. 2007;66(2):158-62.

33. Maillefert JF, Kloppenburg M, Fernandes L, Punzi L, Gunther KP, Martin Mola E, Lohmander LS, Pavelka K, Lopez-Olivo MA, Dougados M, et al. Multilanguage translation and cross-cultural adaptation of the OARSI/OMERACT measure of intermittent and constant osteoarthritis pain (ICOAP). Osteoarthr Cartil. 2009;17(10):1293-6.

34. Herdman M, Gudex C, Lloyd A, Janssen M, Kind P, Parkin D, Bonsel G, Badia $X$. Development and preliminary testing of the new five-level version of EQ5D (EQ-5D-5L). Qual Life Res. 2011;20(10):1727-36.

35. Versteegh M, Vermeulen K, Evers S, de Wit G, Prenger R, Stolk E. Dutch tariff for the five-level version of EQ-5D. Value Health. 2016;19(4):343-52.

36. Rienstra W, Blikman T, Mensink FB, van Raay JJ, Dijkstra B, Bulstra SK, Stevens $M$, van den Akker-Scheek I. The modified painDETECT questionnaire for patients with hip or knee osteoarthritis: translation into Dutch, cross-cultural adaptation and reliability assessment. PLoS One. 2015;10(12):e0146117.

37. Craig CL, Marshall AL, Sjostrom M, Bauman AE, Booth ML, Ainsworth BE, Pratt M, Ekelund U, Yngve A, Sallis JF, et al. International physical activity questionnaire: 12-country reliability and validity. Med Sci Sports Exerc. 2003; 35(8):1381-95.

38. Pham T, van der Heijde D, Altman RD, Anderson JJ, Bellamy N, Hochberg M, Simon L, Strand V, Woodworth T, Dougados M. OMERACT-OARSI initiative: osteoarthritis research society international set of responder criteria for osteoarthritis clinical trials revisited. Osteoarthr Cartil. 2004;12(5):389-99.

39. iMCQ: iMedical Consumption Questionnaire. http://www.imta.nl/ questionnaires. Accessed Oct 2016.

40. Henriksen M, Christensen R, Klokker L, Bartholdy C, Bandak E, Ellegaard K, Boesen MP, Riis RG, Bartels EM, Bliddal H. Evaluation of the benefit of corticosteroid injection before exercise therapy in patients with osteoarthritis of the knee: a randomized clinical trial. JAMA Intern Med. 2015;175(6):923-30.
41. KOOS: Knee injury and Osteoarthritis Outcome Score (KOOS) questionnaire. Assessed October 2016

42. Mills KA, Naylor JM, Eyles JP, Roos EM, Hunter DJ. Examining the minimal important difference of patient-reported outcome measures for individuals with knee osteoarthritis: a model using the knee injury and osteoarthritis outcome score. J Rheumatol. 2016;43(2):395-404.

43. Brinks A, van Rijn RM, Willemsen SP, Bohnen AM, Verhaar JA, Koes BW, Bierma-Zeinstra SM. Corticosteroid injections for greater trochanteric pain syndrome: a randomized controlled trial in primary care. Ann Fam Med. 2011;9(3):226-34.

44. Altman DG. Comparability of randomized groups. J R Stat Soc Ser D-Stat. 1985;34(1):125-36.

45. de Boer MR, Waterlander WE, Kuijper LD, Steenhuis IH, Twisk JW. Testing for baseline differences in randomized controlled trials: an unhealthy research behavior that is hard to eradicate. Int J Behav Nutr Phys Act. 2015;12:4.

46. Rehal S, Morris TP, Fielding K, Carpenter JR, Phillips PP. Non-inferiority trials: are they inferior? A systematic review of reporting in major medical journals. BMJ Open. 2016;6(10):e012594

47. van Middelkoop M, Arden NK, Atchia I, Birrell F, Chao J, Rezende MU, Lambert RG, Ravaud P, Bijlsma JW, Doherty M, et al. The OA trial Bank: meta-analysis of individual patient data from knee and hip osteoarthritis trials show that patients with severe pain exhibit greater benefit from intraarticular glucocorticoids. Osteoarthr Cartil. 2016;24(7):1143-52.

\section{Publisher's Note}

Springer Nature remains neutral with regard to jurisdictional claims in published maps and institutional affiliations.
Ready to submit your research? Choose BMC and benefit from:

- fast, convenient online submission

- thorough peer review by experienced researchers in your field

- rapid publication on acceptance

- support for research data, including large and complex data types

- gold Open Access which fosters wider collaboration and increased citations

- maximum visibility for your research: over $100 \mathrm{M}$ website views per year

At BMC, research is always in progress.

Learn more biomedcentral.com/submissions 